\title{
LAMOST J202629.80+423652.0 is not a symbiotic star
}

\author{
V. Andreoli ${ }^{1}$ and U. Munari ${ }^{2}$ \\ 1 ANS Collaboration, Astronomical Observatory, 36012 Asiago (VI), Italy \\ ${ }^{2}$ INAF Padova Astronomical Observatory, I-36012 Asiago (VI), Italy
}

Received: March 24, 2020; Accepted: April 15, 2020

\begin{abstract}
LAMOST J202629.80+423652.0 has been recently classified as a new symbiotic star containing a long-period Mira, surrounded by dust (Dtype) and displaying in the optical spectra high ionization emission lines, including the Raman-scattered OVI at $6825 \AA$. We have observed LAMOST J202629.80+423652.0 photometrically in the BVRI bands and spectroscopically over the $3500-8000 \AA$ range. We have found it to be a normal G8 IV sub-giant star, deprived of any emission line in its spectrum, and reddened by $E_{B-V}=0.35$ mag. Combining our photometry with data from all-sky patrol surveys, we find LAMOST J202629.80+423652.0 to be non variable, so not pulsating as a Mira. We have compiled from existing sources its spectral energy distribution, extending well into the mid-Infrared, and found it completely dominated by the G8 IV photospheric stellar emission, without any sign of circumstellar dust. We therefore conclude that LAMOST J202629.80+423652.0 is not a symbiotic star, nor it is pulsating or been enshrouded in dust.
\end{abstract}

Key words: binaries: symbiotic - surveys: LAMOST

\section{Introduction}

Symbiotic stars (SySt) are binary systems composed by a red giant star (RG) that transfers material to a degenerate companion which can be either a white dwarf (WD) or a neutron star (NS). SySt are broadly divided into two major groups (see the recent review by Munari 2019): those accreting-only whose optical spectra are dominated by the RG with no or weak emission lines, and the burning-type displaying a strong nebular continuum and a rich emission line spectrum: they originate in the wind of the RG largely ionized by the very hot and luminous WD companion that is nuclearly burning at the surface in stable conditions. The accreting-only SySt usually requires satellite UV/X-ray observations to be discovered (cf. the prototype SU Lyn, Mukai et al. 2016), while the burning StSy can easily be discovered through the whole Galaxy and the Local Group thanks to the prominent emission lines. In about $20 \%$ of the known SySt (see recent catalogs of symbiotic stars by Belczyński et al. 2000, and Akras et al. 2019), the cool giant pulsates as a Mira and this usually comes also with the 
presence of warm circumstellar dust ( 500-1000 K), leading to a classification as D-type according to the scheme introduced by Allen (1982).

Li et al. (2015) have announced the discovery of two new SySt during the LAMOST all-sky spectroscopic survey. LAMOST is a $4 \mathrm{~m}$ Schmidt telescope located in Xinglong (China), feeding light to 16 spectrographs via 4000 optical fibers. The spectrum presented by Li et al. for one of these two new SySt, namely LAMOST J202629.80+423652.0 (hereafter LM), looks weird and we suspected to be the result of problematic sky subtraction, so decided to investigate the matter by acquiring new spectra and supplementing them with acquisition of BVRI photometry. We report in this paper the results of these observations.

\section{Observations}

\subsection{Spectroscopy}

Low resolution spectra of LM have been obtained with the $1.22 \mathrm{~m}$ telescope operated in Asiago by the University of Padova. A Boller \& Chivens spectrograph is attached to the Cassegrain focus feeding light to an ANDOR iDus DU44OA camera, that houses a back-illuminated E2V 42-100 CCD (2048 X 512 array, $13.5 \mu \mathrm{m}$ pixel). A $300 \mathrm{ln} \mathrm{mm}^{-1}$ grating blazed at $5000 \AA$ was adopted, allowing to cover the $3300-8000 \AA$ range at $2.31 \AA$ /pix dispersion. Data reduction was carried out in IRAF and involved the usual steps on bias and dark removal, flat-fielding, variance-weighted spectrum tracing, sky subtraction, wavelength calibration via FeHeAr lamp, heliocentric correction and flux calibration (via observations of the nearby spectrophotometric standard HR 7867). LM has been observed twice on Dec 6 and 14, 2019, exposing for 16min on both occasions. The spectra look identical and their average is plotted in Figure 1. A comparison is there provided with HD 188512, a template for the G8 IV spectral class according to Yamashita et al. (1977), observed with exactly the same telescope setup as for LM, and selected from the Asiago Spectral Database (U. Munari, in preparation).

\subsection{Photometry}

Optical photometry of LM in the Landolt (1992) photometric system has been obtained with a Richey-Chretien $0.4 \mathrm{~m} \mathrm{f} / 8$ telescope located in Monte Baldo (Verona, Italy), equipped with a Moravian G4 CCD camera $(36 \times 36 \mathrm{~mm}$ active area, $12 \mu \mathrm{m}$ size) and B,V,R,I photometric filters from Astrodon. The B filter is of the new version, corrected for the red-leak of its original version (see Munari and Moretti 2012). The photometry of LM that we have collected in five distinct nights is presented in Table 1.

Data reduction has involved all the usual steps for bias, dark, flat with calibration images collected during the same observing nights. We adopted aperture 


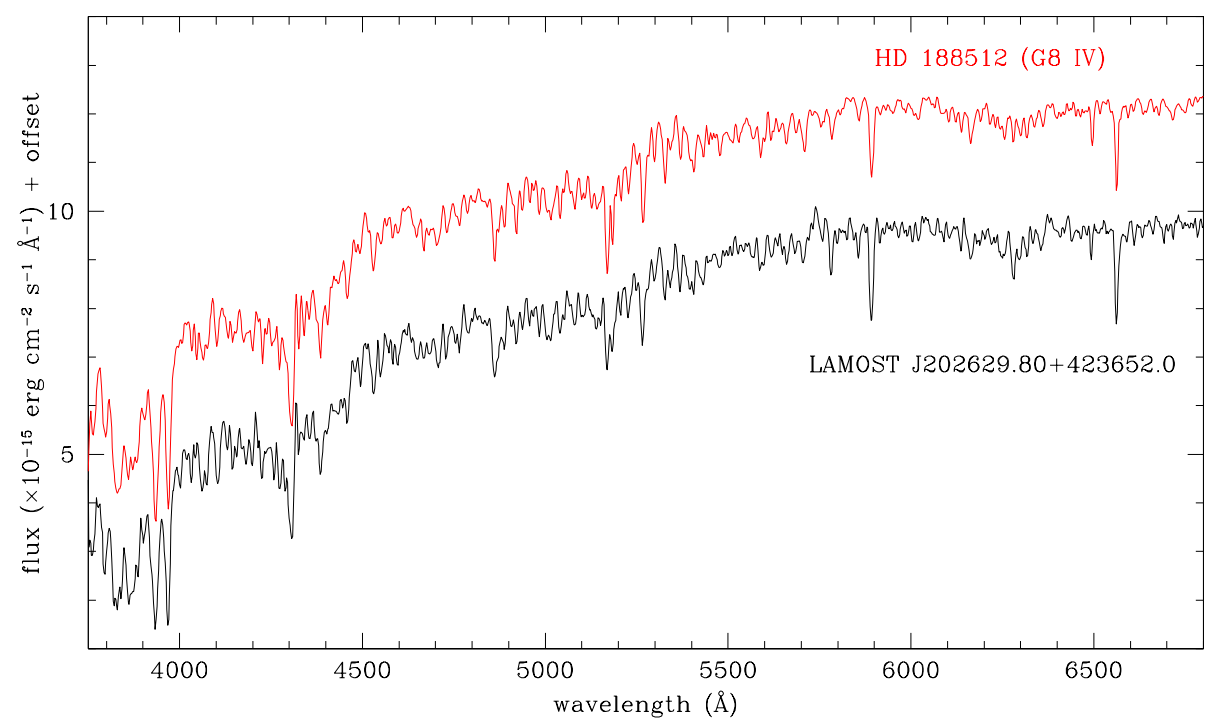

Figure 1. Our spectrum of LAMOST J202629.80+423652.0 compared to that of HD 188512 (+offset), template for the G8 IV spectral type.

photometry because the sparse field around LM did not required PSF-fitting procedures. The transformation from the local to the Landolt standard system was carried out via color equations calibrated on a photometric sequence recorded on the same frames as LM:

$$
\begin{aligned}
V & =v+\alpha_{v} \times(v-i)+\gamma_{v} \\
B-V & =\beta_{b v} \times(b-v)+\delta_{b v} \\
V-R & =\beta_{v r} \times(v-r)+\delta_{v r} \\
V-I & =\beta_{v i} \times(v-i)+\delta_{v i}
\end{aligned}
$$

The local photometric sequence has been extracted from APASS DR8 survey (Henden and Munari 2014), ported to the Landolt system via the transformations calibrated by Munari et al. (2014). The errors quoted in Table 1 are the quadratic sum of the Poissonian and the transformation contributions.

\section{Results}

\subsection{A normal spectral appearance}

The spectrum of LM presented in Figure 1 is that of a normal, sub-giant star of the G8 IV spectral type, abundant in the Solar Neighborhood as indicated by Gaia DR2 stellar census (Gaia Collaboration 2018). No Mira is known to 
Table 1. Our BVRI photometry (on the Landolt system of equatorial standards) of LAMOST J202629.80+423652.0.

\begin{tabular}{ccccc}
\hline \hline obs. date & $\mathrm{B}$ & $\mathrm{V}$ & $\mathrm{R}$ & $\mathrm{I}$ \\
\hline $2019-12-07.8957$ & & $14.439 \pm 0.009$ & $13.766 \pm 0.010$ & $13.041 \pm 0.014$ \\
$2019-12-14.8155$ & & $14.456 \pm 0.006$ & $13.773 \pm 0.007$ & $13.119 \pm 0.016$ \\
$2020-01-08.8245$ & $15.629 \pm 0.032$ & $14.454 \pm 0.011$ & $13.760 \pm 0.010$ & $13.100 \pm 0.015$ \\
$2020-01-09.8242$ & $15.603 \pm 0.061$ & $14.478 \pm 0.029$ & $13.737 \pm 0.017$ & $13.076 \pm 0.025$ \\
$2020-01-16.7712$ & $15.580 \pm 0.019$ & $14.454 \pm 0.006$ & & \\
& & & & \\
weighted mean & $15.610 \pm 0.020$ & $14.463 \pm 0.004$ & $13.755 \pm 0.005$ & $13.084 \pm 0.008$ \\
\hline \hline
\end{tabular}

have such an early spectral type (cf. VSX database ${ }^{1}$ ). In our spectrum of LM no emission line is visible.

Li et al. (2015) report the presence of strong $\mathrm{H} \alpha, \mathrm{H} \beta$ and [OIII] emission lines in their spectrum of LM. They come actually from the sky-background emission originating from the huge HII region IC 1318 associated to nearby $\gamma$ $\mathrm{Cyg}\left(10^{\circ}\right.$ angular extent). In Figure 2 we present the sky-background at 15 arcsec away from LM as recorded on our spectrum. It looks identical to the spectrum attributed to LM by Li et al. (2015).

Li et al. (2015) also report about the detection in their spectrum of LM of Raman scattered OVI lines at 6830 and $7088 \AA$ (Schmid 1989). These are actually normal $\mathrm{OH}$ lines forming in the Earth's atmosphere, frequently used for wavelength calibration of high resolution spectra at far-red wavelengths (cf. Osterbrock et al. 1996). In Figure 3 we plot the far-red portion of the sky-background at 15 arcsec distance from LM taken from our spectrum of the program star, with superimposed the identification of Meinel rotation-vibration bands of $\mathrm{OH}$.

We therefore conclude this section noting that nothing in the spectrum of LM supports a classification as a SySt: all lines and features seen in emission by Li et al. (2015) seems actually due to inaccurate handling of sky-background subtractions.

\subsection{No photometric variability}

Li et al. (2015) also classified LM as a pulsating Mira. Our photometry in Table 1 extends over too short a time interval to draw any conclusion about a possible variability affecting LM. To investigate the matter futher, we retrieved the patrol photometry of LM collected by ASASSN (Shappee et al. 2014, Kochanek et al. 2017) and ZTF all-sky patrol surveys (Masci et al. 2019, Bellm et al. 2019). The ASASSN and ZTF photometry in the $g^{\prime}, r^{\prime}$, and $V$ bands is plotted in Figure 4. It extends from 2015 to 2019 . No variability is present in excess of the

\footnotetext{
${ }^{1}$ https://www.aavso.org/vsx/
} 


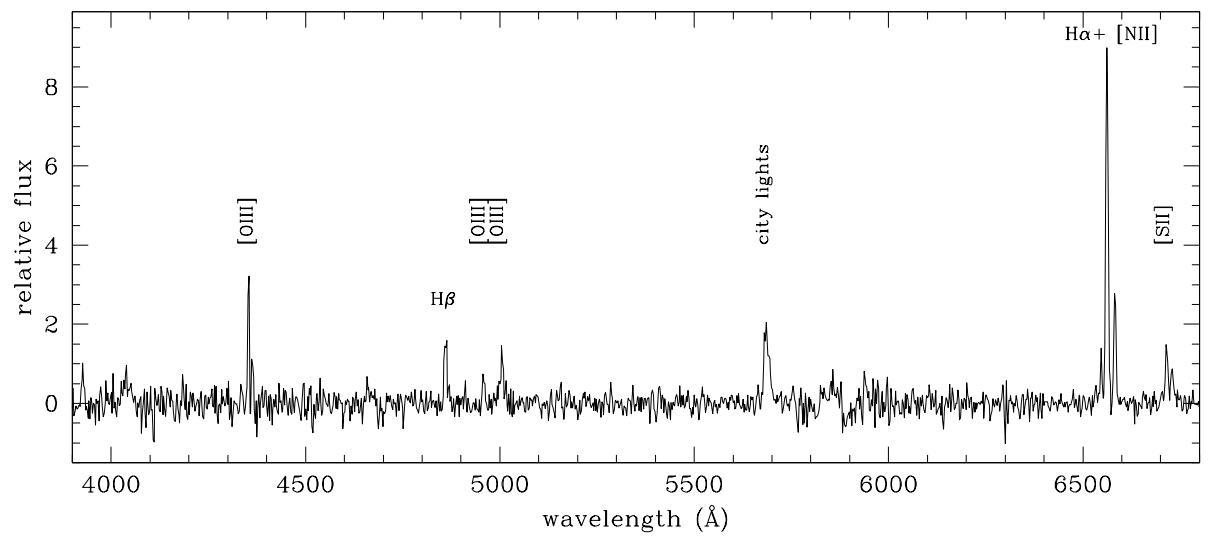

Figure 2. Emission lines from the sky-background around LAMOST J202629.80+423652.0 in our spectrum, due to diffuse emission from the HII region around $\gamma$ Cyg.

observational noise, and this precludes the classification reported by $\mathrm{Li}$ et al. (2015) of LM as a Mira.

\subsection{No circumstellar dust}

Li et al. (2015) attributed a D-type classification to LM, i.e. the presence of warm dust around it. As illustrated by Allen (1982) the excess emission from such warm dust $(500-1000 \mathrm{~K})$ is already evident in the $H$ band $(1.6 \mu \mathrm{m})$ and become prominent in the $K$ band $(2.2 \mu \mathrm{m})$. To investigate the spectral energy distribution (SED) of LM, we take advantage of the absence of photometric variability (established in the previous section) that allows to combine non-simultaneous data gathered by different surveys over the whole wavelength range. The SED for LM built from our BVRI observations (Table 1), 2MASS $\mathrm{JHK}_{s}$ and AllWISE $\mathrm{W}_{1} \mathrm{~W}_{2} \mathrm{~W}_{3}$ data is presented in Figure 5, together with the reference SED for a typical G8 IV field star using colors from Fitzgerald (1970), Cousins (1976), and Koornneef et al. (1983). The two become a fine match once a correction for $E_{B-V}=0.35$ reddening is applied to LM. In conclusion, the SEDs in Figure 5 excludes the presence in LM of any dust hotter that $\sim 100 \mathrm{~K}$ (constrain set by $\mathrm{W}_{3}$ ).

\subsection{Fundamental parameters}

The GAIA second release (GAIA-DR2) lists a parallax $\pi=0.761 \pm 0.018$ (mas) for LM. The fractional error is less than $20 \%$ and therefore is straightforward to derived the distance to $\mathrm{LM}$ as $\mathrm{d}=1.31 \mathrm{kpc}$ by direct inversion of the Gaia parallax. At such a distance along the line of sight to LM, the Bayestar 3D Galactic 


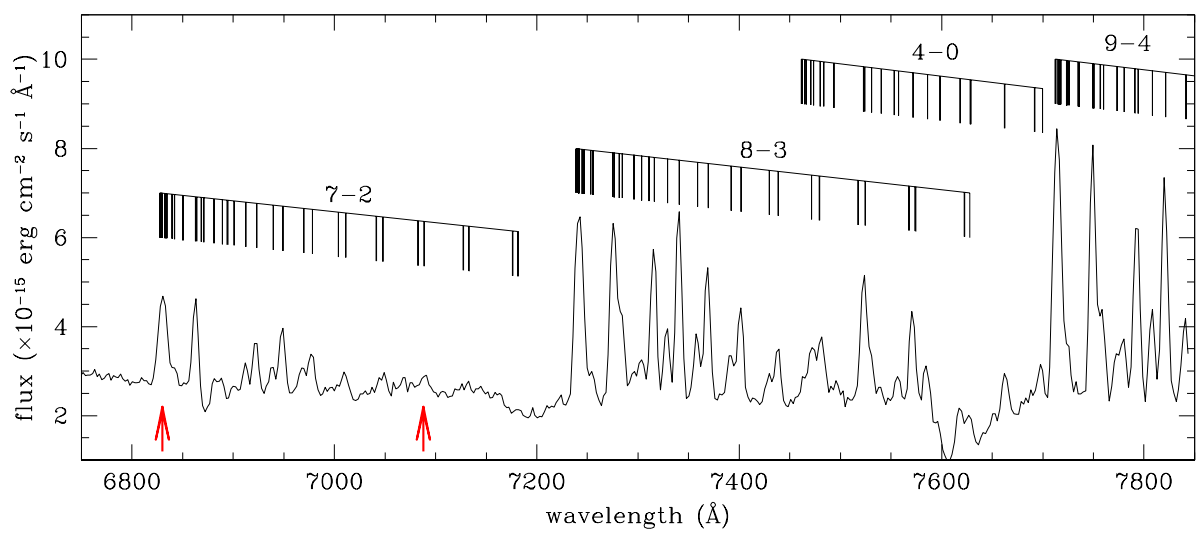

Figure 3. Emission in the far-red of the sky-background around LAMOST J202629.80+423652.0 in our spectrum, highlighting the Earth's OH emission (with Meinel rotation-vibration bands identified). The red arrows points to telluric $\mathrm{OH}$ lines labelled by Li et al. (2015) as due to Raman scattered OVI 6825, $7088 \AA$.

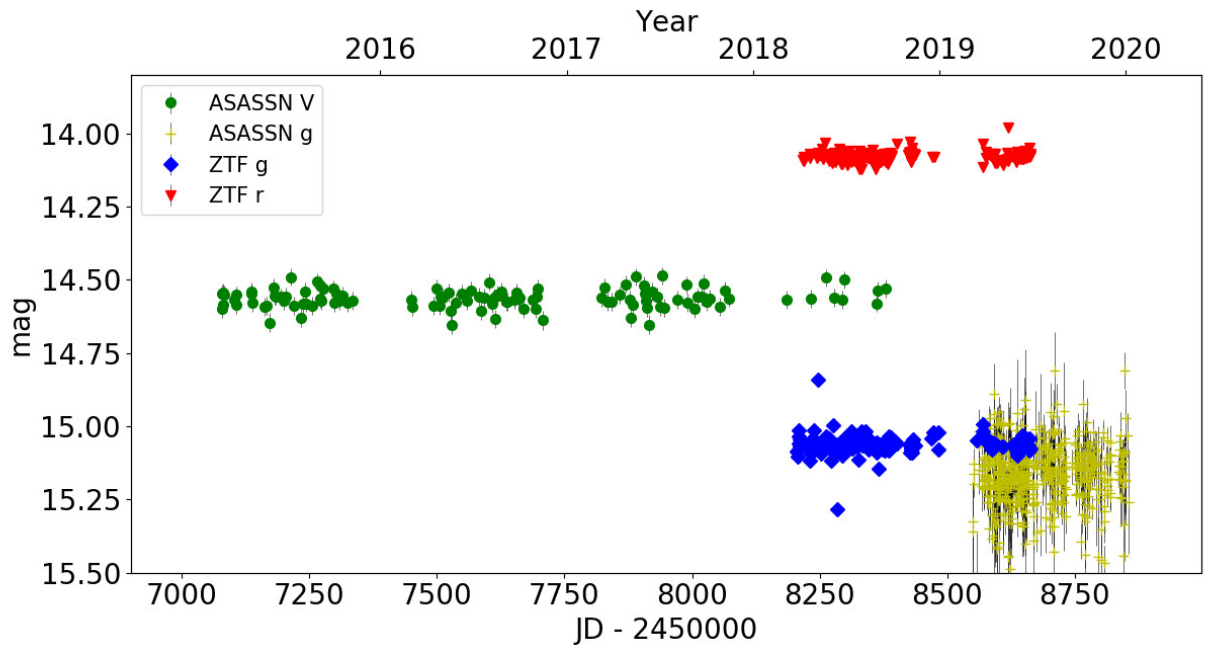

Figure 4. Photometric data of LAMOST J202629.80+423652.0 obtained by ASAS-SN and ZTF sky patrol surveys. 


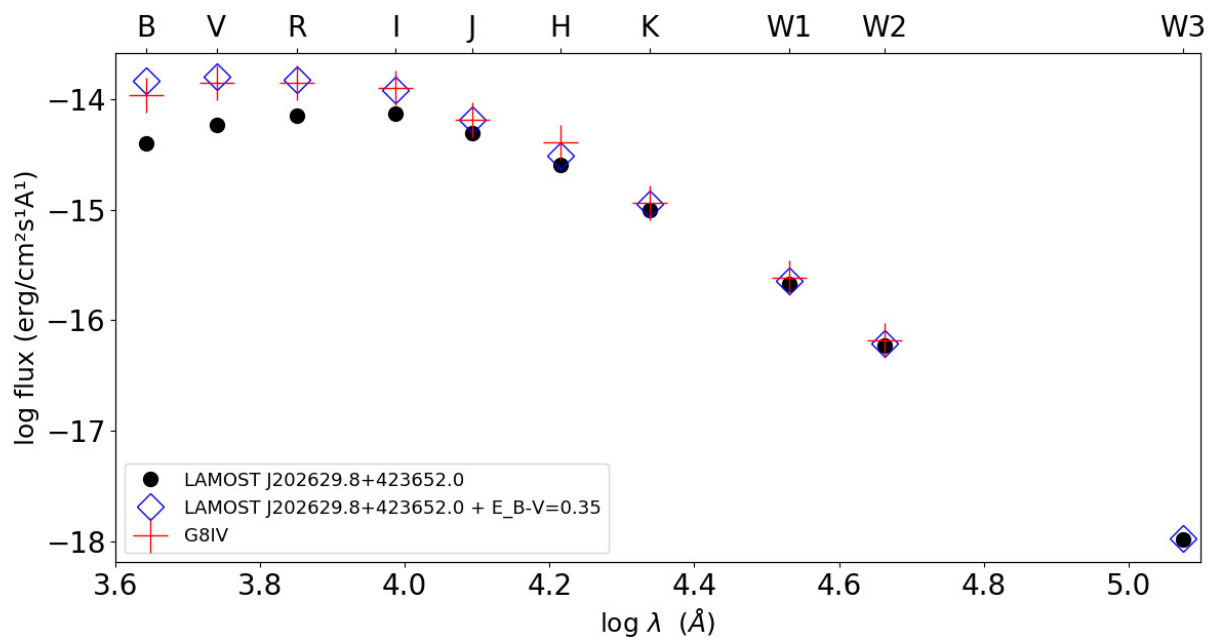

Figure 5. Spectral energy distribution of LAMOST J202629.80+423652.0 as observed (black dots), and after correction for $E_{B-V}=0.35$ reddening (blue diamonds). The red crosses illustrate the intrinsic energy distribution of a G8 IV star from tabular data.

extinction map (Green et al. 2019) lists a reddening of $E(g-r)=0.41$, that transforms to $E(B-V)_{0}=0.35$ for a standard $R_{V}=3.1$ interstellar reddening law following the relations for G-type stars by Fiorucci and Munari (2003). This is in excellent agreement with the value of the reddening necessary to bring the SED of LM in agreement with that of a typical G8 IV star in Figure 5. With such a reddening and distance, the mean $V$-mag value in Table 1 transforms to an absolute magnitude for LM of $M(V)=2.78$. This is in fine agreement with the absolute magnitudes reported by Sowell et al. (2007) that list $\mathrm{M}(\mathrm{V})=5.50$, 3.20 and 1.35 for G8 stars of luminosity class V, IV and III, respectively. Accordingt to tabular values in Straizys (1992), such a star is characterized by a temperature of $5100 \mathrm{~K}$, a luminosity of $4.4 \mathrm{~L}_{\odot}$ and a radius of $2.7 \mathrm{R}_{\odot}$.

\section{Conclusions}

We have obtained new photometric and spectroscopic observations of LAMOST J202629.80+423652.0 that combined with literature data shows it to be a normal G8 IV sub-giant star, and not a symbiotic star. Such a classification by Li et al. (2015) appears due to inaccurate sky-background subtraction to their spectra.

\section{References}

Akras, S., Guzman-Ramirez, L., Leal-Ferreira, M.L., Ramos-Larios, G., A census of symbiotic stars in the 2MASS, WISE, and GAIA suvey, 2019, Astrophys. J., Suppl. Ser., 240, p.21, DOI: 10.3847/1538-4365/aaf88c 
Allen, D.A., The Nature of Symbiotic Stars, 2000, ed. M. Friedjung and R. Viotti, IAU Colloq, 1982, 95, $20+310 \mathrm{p}$

Belczyński, K., Mikoajewska, J., Munari, U., Ivison, R., Friedjung, M., A catalogue of symbiotic stars, 2000, Astron. Astrophys., Suppl., 146, p.407, DOI: 10.1051/aas:2000280

Bellm, E.C., et al., The Zwicky Transient Facility: System Overview, Performance, and First Results, 2019, Publ. Astron. Soc. Pac., 131, p.018002, DOI: $10.1088 / 1538-3873 /$ aaecbe

Cousins, K.W.J., VRI standards in the E regions, 1976, Mem. Royal Astron. Soc., 81, p.25

Fiorucci, M., Munari, U., The Asiago Database on Photometric Systems (ADPS). II. Band and reddening parameters, 2003, Astron. Astrophys., 401, p.781, DOI: 10.1051/0004-6361:20030075

Fitzgerald, M. Pim., The Intrinsic Colours of Stars and Two-Colour Reddening Lines, 1970, Astron. Astrophys., 4, p.234

Gaia Collaboration., Mignard, F., Klioner, S.A., Lindegren, L., Hernndez, J., Bastian, U., Bombrun, A., Hobbs, D., Lammers, U., Michalik, D., RamosLerate, M., Biermann, M., Fernndez-Hernndez, J., Geyer, R., Hilger, T., Siddiqui, H.I., Babusiaux, C., Barache, C., Lambert, S., and more., Gaia Data Release 2. The celestial reference frame, 2018, Astron. Astrophys., 616, p.A14, DOI: $10.1051 / 0004-6361 / 201832916$

Green, Gregory M., Schlafly, Edward., Zucker, Catherine., Speagle, Joshua S., Finkbeiner, Douglas., A 3D Dust Map Based on Gaia, Pan-STARRS 1, and 2MASS, 2019, Astrophys. J., 881, p.93, DOI: 10.3847/1538-4357/ab5362

Henden, A., Munari, U., The APASS all-sky, multi-epoch BVgri photometric survey, 2014, Contrib. Astron. Obs. Skalnaté Pleso., 43, p.518

Kochanek, C.S., Shappee, B.J., Stanek, K.Z., Holoien, T.W.-S., Thompson, Todd A., Prieto, J.L., Dong, Subo, Shields, J.V., Will, D., Britt, C., Perzanowski, D., Pojmaski, G., The All-Sky Automated Survey for Supernovae (ASAS-SN) Light Curve Server v1.0, 2017, Publ. Astron. Soc. Pac., 129, p.104502, DOI: $10.1088 / 1538-3873 / a a 80 d 9$

Koornneef, J., Near-infrared photometry. II. Intrinsic colours and the absolute calibration from one to five micron, 1983, Astron. Astrophys., 500, p.247

Landolt, Arlo U., UBVRI Photometric Standard Stars in the Magnitude Range 11.5 \&lt; V \&lt; 16.0 Around the Celestial Equator, 1992, Astron. J., 104, p.340, DOI: $10.1086 / 116242$

Li, Jiao., Mikolajewska, Joanna., Chen, Xue-Fei., Luo, A. -Li., RebassaMansergas, Alberto., Hou, Yong-Hui., Wang, Yue-Fei., Wu, Yue., Yang, Ming., Zhang, Yong., Han, Zhan-Wen., The first symbiotic stars from the LAMOST survey, 2015, RAA., 15, p.1332, DOI: 10.1088/1674-4527/15/8/016 
Masci, F. J. et al., The Zwicky Transient Facility: Data Processing, Products, and Archive, 2019, Publ. Astron. Soc. Pac., 131, p.018003, DOI: $10.1088 / 1538-3873 /$ aae8ac

Mukai, K. et al., SU Lyncis, a hard X-ray bright M giant: clues point to a large hidden population of symbiotic stars, 2016, Mon. Not. R. Astron. Soc., 461, p.L1, DOI: $10.1093 / \mathrm{mnrasl} / \mathrm{slw} 087$

Munari, U., in The Impact of Binary Stars on Stellar Evolution, G. Beccari and H.M.J. Boffin eds, 2019, Cambridge Univ. Press., p.77, arXiv:190901389, DOI: $10.1017 / 9781108553070$

Munari, U., Moretti, S., Characterizing the Photometric Response of the ANS Collaboration Monitoring Program, 2012, Baltic Astronomy., 21 p.22, DOI: 10.1515/astro-2017-0354

Munari, U., Henden, A., Frigo, A., APASS discovery and characterization of 180 variable stars in Aquarius, 2014, JAD., 20, p.4

Osterbrock, D.E., Fulbright, J.P., Martel, A.R., Keane, M.J., Trager, S.C., Basri, G., Night-Sky High-Resolution Spectral Atlas of OH and O2 Emission Lines for Echelle Spectrograph Wavelength Calibration, 1996, Publ. Astron. Soc. Pac., 108, p.277

Sowell, J. R., et al., H-R Diagrams Based on the HD Stars in the Michigan Spectral Catalogue and the Hipparcos Catalog, Astron. J., 2007, 134, p.1089, DOI: $10.1086 / 520060$

Schmid, H.M., Identification of the emission bands at 6830, $7088 \AA$, 1989, Astron. Astrophys., 211, p.L31

Shappee, B.J., Prieto, J.L., Grupe, D., Kochanek, C.S., Stanek, K.Z., De Rosa, G., Mathur, S., Zu, Y., Peterson, B.M., Pogge, R.W., Komossa, S., Im, M., Jencson, J., Holoien, T.W.-S., Basu, U., Beacom, J.F., Szczygie, D.M., Brimacombe, J., Adams, S., Campillay, A., et al., The Man behind the Curtain: X-Rays Drive the UV through NIR Variability in the 2013 Active Galactic Nucleus Outburst in NGC 2617, 2014, The Astronomical Journal., 788, p.48, DOI: $10.1088 / 0004-637 \mathrm{X} / 788 / 1 / 48$

Straižys, V., Multicolor stellar photometry, 1992, Tucson : Pachart Pub. House, c1992

Yamashita, Y., Nariai, K., An Atlas of representative stellar spectra, 1977, University of Tokio Press 